\title{
New Prediction for Extended Targets With Random Matrices
}

\author{
Karl Granström and U. Orguner
}

Linköping University Post Print

Tweet

N.B.: When citing this work, cite the original article.

Karl Granström and U. Orguner, New Prediction for Extended Targets With Random Matrices, 2014, IEEE Transactions on Aerospace and Electronic Systems, (50), 2, 1577-1589.

http://dx.doi.org/10.1109/TAES.2014.120211

C2014 IEEE. Personal use of this material is permitted. However, permission to reprint/republish this material for advertising or promotional purposes or for creating new collective works for resale or redistribution to servers or lists, or to reuse any copyrighted component of this work in other works must be obtained from the IEEE.

http://ieeexplore.ieee.org/

Postprint available at: Linköping University Electronic Press

http://urn.kb.se/resolve?urn=urn:nbn:se:liu:diva-112656 


\title{
A New Prediction for Extended Targets with Random Matrices
}

\author{
Karl Granström, Member, IEEE, and Umut Orguner, Member, IEEE
}

\begin{abstract}
This paper presents a new prediction update for extended targets whose extensions are modeled as random matrices. The prediction is based on several minimizations of the Kullback-Leibler divergence and allows for a kinematic state dependent transformation of the target extension. The results show that the extension prediction is a significant improvement over the previous work carried out on the topic.
\end{abstract}

Index Terms-Extended target, random matrix, KullbackLeibler divergence, inverse Wishart, Wishart, generalized Beta.

\section{INTRODUCTION}

Extended targets are targets that potentially give rise to more than one measurement per time step, in contrast to standard targets that give rise to at most one measurement per time step, see e.g. [1]. The multiple measurements per time step raise interesting possibilities to estimate the target's extension, i.e. the shape and size. Several extended target models have been proposed in the literature, see e.g. [2]-[8] and the references therein.

In the extended target model proposed by Koch et al. [7], [8], the target extension is modeled as an ellipse, and it is represented by a positive definite matrix called extension matrix. The extended target originated measurements are modeled as being (approximately) Gaussian distributed, with covariance related to the extension matrix. Following a Bayesian methodology, the extension matrix is modeled to be a random variable ${ }^{1}$ that is inverse Wishart distributed. The overall extended target state is defined as the combination of the extension matrix and the usual kinematical state vector. The parameters of the kinematical state density, and the extension's inverse Wishart density, are updated in a Bayesian recursion, which consists of prediction (time update) and correction (measurement update).

The focus in this paper is on the prediction update of extended targets within the random matrix framework. In early work, see [7], [8], the extension matrix' prediction was based on simple heuristics which increase the extension's covariance, while keeping the expected value constant. Koch also discusses the use of a Wishart transition density for the extension state [7], see also [9], [10]. In this paper we generalize this idea by including the possibility of a kinematic state dependent

Karl Granström is with the Department of Electrical Engineering, Division of Automatic Control, Linköping University, Linköping, SE-581 83, Sweden, e-mail: karleisy.liu.se.

Umut Orguner is with the Department of Electrical and Electronics Engineering, Middle East Technical University, 06531, Ankara, Turkey, e-mail: umut deee.metu.edu.tr.

${ }^{1}$ Hence we refer to the model [7], [8] as the the random matrix framework. transformation of the extension. This would, for example, be useful when the target extension rotates during a coordinatedturn, a situation which appears very frequently in air traffic control applications. In order to derive a Bayesian prediction update for the extension, minimizations of the KullbackLeibler divergence are used to approximate densities. This methodology enables us to make well-defined approximations when the original density and its approximation have different numbers of parameters.

The rest of the paper is organized as follows. In Section II we give a brief introduction to the random matrix framework, and present the approaches to prediction given in [7]-[10]. Section III presents a problem formulation and defines the main aim of the study. In Section IV, we give results that are used in the derivation of the main result, which is a new prediction update presented in Section V. The merits of the new update are illustrated in simulations, with comparisons to previous methods in Section VI. Concluding remarks are given in Section VII.

\section{THE RANDOM MATRIX FRAMEWORK}

We use the following notation:

- $\mathbb{R}^{n}$ is the set of real column vectors of length $n, \mathbb{S}_{++}^{n}$ is the set of symmetric positive definite $n \times n$ matrices, and $\mathbb{S}_{+}^{n}$ is the set of symmetric positive semi-definite $n \times n$ matrices.

- $\mathcal{N}(\mathbf{x} ; \mathbf{m}, P)$ denotes a multi-variate Gaussian probability density function (pdf) defined over the vector $\mathbf{x} \in \mathbb{R}^{n_{x}}$ with mean vector $\mathbf{m} \in \mathbb{R}^{n_{x}}$, and covariance matrix $P \in \mathbb{S}_{+}^{n_{x}}$,

$$
\mathcal{N}(\mathbf{x} ; \mathbf{m}, P)=\frac{e^{-\frac{1}{2}(\mathbf{x}-\mathbf{m})^{\mathrm{T}} P^{-1}(\mathbf{x}-\mathbf{m})}}{(2 \pi)^{\frac{n_{x}}{2}}|P|^{\frac{1}{2}}},
$$

where $|\cdot|$ is the matrix determinant function.

- $\mathcal{I W}_{d}(X ; v, V)$ denotes an inverse Wishart pdf defined over the matrix $X \in \mathbb{S}_{++}^{d}$ with scalar degrees of freedom $v>2 d$ and parameter matrix $V \in \mathbb{S}_{++}^{d},[11$, Definition 3.4.1]

$\mathcal{I W}_{d}(X ; \nu, V)=\frac{2^{-\frac{\nu-d-1}{2}}|V|^{\frac{\nu-d-1}{2}}}{\Gamma_{d}\left(\frac{\nu-d-1}{2}\right)|X|^{\frac{\nu}{2}}} \operatorname{etr}\left(-\frac{1}{2} X^{-1} V\right)$

where $\operatorname{etr}(\cdot)=\exp (\operatorname{Tr}(\cdot))$ is exponential of the matrix trace, and $\Gamma_{d}(\cdot)$ is the multivariate gamma function. The multivariate gamma function can be expressed as a product of ordinary gamma functions, see e.g. [11, Theorem 1.4.1]. 
- $\mathcal{W}_{d}(X ; w, W)$ denotes a Wishart pdf defined over the matrix $X \in \mathbb{S}_{++}^{d}$ with scalar degrees of freedom $w>$ $d-1$ and parameter matrix $W \in \mathbb{S}_{++}^{d}$, [11, Definition 3.2.1]

$$
\mathcal{W}_{d}(X ; w, W)=\frac{2^{-\frac{w d}{2}}|X|^{\frac{w-d-1}{2}}}{\Gamma_{d}\left(\frac{w}{2}\right)|W|^{\frac{n}{2}}} \operatorname{etr}\left(-\frac{1}{2} W^{-1} X\right) .
$$

- $\mathcal{G B}_{d}^{I I}(X ; a, b, \Omega, \Psi)$ denotes a generalized matrix-variate beta type-II pdf defined over the matrix $X \in \mathbb{S}_{++}^{d}$, with scalar parameters $a>\frac{d-1}{2}, b>\frac{d-1}{2}$ and matrix parameters $\Omega \in \mathbb{S}_{++}^{d}, \Psi \in \mathbb{S}_{+}^{d}$, [11, Definition 5.2.4]

$$
\mathcal{G B}_{d}^{I I}(X ; a, b, \Omega, \Psi)=\frac{|X-\Psi|^{a-\frac{d+1}{2}}|\Omega+X|^{-(a+b)}}{\beta_{d}(a, b)|\Omega+\Psi|^{-b}},
$$

where $(X-\Psi) \in \mathbb{S}_{++}^{d}$ and $(\Omega-\Psi) \in \mathbb{S}_{++}^{d}$.

- $\mathbf{0}_{d}$ is an all zero $d \times d$ matrix, and $\mathbf{I}_{d}$ is a $d \times d$ identity matrix.

Let $\xi_{k}$ be the extended target state at time $t_{k}$, and let $\mathbf{Z}^{k}$ denote the set of all measurements up to and including time $t_{k}$. The random matrix framework [7], [8] defines the extended target state $\xi_{k}=\left(\mathbf{x}_{k}, X_{k}\right)$ as the combination of a kinematical state $\mathbf{x}_{k} \in \mathbb{R}^{n_{x}}$ and an extension state $X_{k} \in \mathbb{S}_{++}^{d}$. The kinematical state $\mathbf{x}_{k}$ contains states related to target kinematics, such as position, velocity and heading, while the extension state $X_{k}$ is a random matrix representing the target extent. The posterior pdf of the extended target state $\xi_{k}$, conditioned on $\mathbf{Z}^{k}$, is modeled as Gaussian inverse Wishart (GIW) distributed [8]

$$
\begin{aligned}
p\left(\xi_{k} \mid \mathbf{Z}^{k}\right) & =p\left(\mathbf{x}_{k} \mid X_{k}, \mathbf{Z}^{k}\right) p\left(X_{k} \mid \mathbf{Z}^{k}\right) \\
& \approx p\left(\mathbf{x}_{k} \mid \mathbf{Z}^{k}\right) p\left(X_{k} \mid \mathbf{Z}^{k}\right) \\
& =\mathcal{N}\left(\mathbf{x}_{k} ; m_{k \mid k}, P_{k \mid k}\right) \mathcal{I} \mathcal{W}_{d}\left(X_{k} ; \nu_{k \mid k}, V_{k \mid k}\right) .
\end{aligned}
$$

This density approximates the kinematical and extension states as independent, however, as noted in [8], the measurement update step "provides for the interdependency between kinematics and extension estimation." The random matrix framework limits the extended targets to be shaped as ellipses, however the ellipse shape is applicable to many real scenarios in which the target and sensor geometry is such that the target measurements resemble a cluster of detections, rather than a geometric structure (or for that matter a single detection).

Note that in a Bayesian state estimation recursion, we (typically) want the predicted pdf $p\left(\xi_{k+1} \mid \mathbf{Z}^{k}\right)$ to be of the same functional form as the posterior pdf $p\left(\xi_{k} \mid \mathbf{Z}^{k}\right)$. For a GIW distributed extended target $(5 \mathrm{c})$, this corresponds to obtaining the parameters $m_{k+1 \mid k}, P_{k+1 \mid k}, \nu_{k+1 \mid k}$, and $V_{k+1 \mid k}$ of the distribution

$$
\begin{aligned}
p\left(\xi_{k+1} \mid \mathbf{Z}^{k}\right)= & \mathcal{N}\left(\mathbf{x}_{k+1} ; m_{k+1 \mid k}, P_{k+1 \mid k}\right) \\
& \times \mathcal{I} \mathcal{W}_{d}\left(X_{k+1} ; \nu_{k+1 \mid k}, V_{k+1 \mid k}\right) .
\end{aligned}
$$

In previous work, see [7], [8], the kinematical state $\mathbf{x}_{k}$ is predicted using the Kalman filter prediction [12]. The extension state prediction is based on simple heuristics. Under the assumption that "the extension does not tend to change over time" [8], the inverse Wishart parameters are predicted such that $\mathrm{E}\left[X_{k+1}\right]=\mathrm{E}\left[X_{k}\right]$ and $\operatorname{Cov}\left(X_{k+1}\right)>\operatorname{Cov}\left(X_{k}\right)$. The following prediction update is used in [8],

$$
\begin{aligned}
\nu_{k+1 \mid k} & =2 d+4+e^{-T / \tau}\left(\nu_{k \mid k}-2 d-4\right), \\
V_{k+1 \mid k} & =\frac{\nu_{k+1 \mid k}-2 d-2}{\nu_{k \mid k}-2 d-2} V_{k \mid k},
\end{aligned}
$$

where $T$ is the prediction time and $\tau$ is a design parameter. Note that (7a) is a minor modification of the prediction $\nu_{k+1 \mid k}=e^{-T / \tau} \nu_{k \mid k}$, which is used in [7]. The modification ensures that the expected value and covariance of $X_{k}$ always are well-defined.

In addition to presenting the prediction update given above, in [7] Koch also discusses using a Wishart extension transition density,

$$
p\left(X_{k+1} \mid X_{k}\right)=\mathcal{W}_{d}\left(X_{k+1} ; n_{k+1}, \frac{X_{k}}{n_{k+1}}\right) .
$$

This transition density is used in [9]. A modified version of (8) is suggested in [10],

$$
p\left(X_{k+1} \mid X_{k}\right)=\mathcal{W}_{d}\left(X_{k+1} ; \delta_{k}, A_{k} X_{k} A_{k}^{\mathrm{T}}\right),
$$

where the $d \times d$ matrix $A_{k}$ describes the extension time evolution, e.g. rotation or scaling of the extension.

The contribution of this paper is a further generalization of the idea to use a Wishart transition density. The presented prediction method allows extension transformations that are functions of the kinematical state.

\section{PROBLEM FORMULATION}

The state transition density $p\left(\xi_{k+1} \mid \xi_{k}\right)$ describes the time evolution of the extended target state from time $t_{k}$ to time $t_{k+1}$. In Bayesian state estimation, the prediction step consists of solving the integral

$$
p\left(\xi_{k+1} \mid \mathbf{Z}^{k}\right)=\int p\left(\xi_{k+1} \mid \xi_{k}\right) p\left(\xi_{k} \mid \mathbf{Z}^{k}\right) \mathrm{d} \xi_{k} .
$$

The transition density can be expressed as [7]

$$
p\left(\xi_{k+1} \mid \xi_{k}\right)=p\left(\mathbf{x}_{k+1} \mid X_{k+1}, \mathbf{x}_{k}\right) p\left(X_{k+1} \mid \mathbf{x}_{k}, X_{k}\right) .
$$

To obtain practical tractability of the prediction update, some assumptions are required. Previous work [7], [8], [10] assumes that the extension time evolution is independent of the kinematical state,

$$
p\left(X_{k+1} \mid \mathbf{x}_{k}, X_{k}\right)=p\left(X_{k+1} \mid X_{k}\right) .
$$

This assumption simplifies extension prediction considerably, and the assumption holds, e.g., when the target performs constant velocity or constant acceleration motion. However, during a constant or variable turn-rate maneuver the assumption does not hold. In this case the extension (typically) rotates during the maneuver, where the rotation is a function of the turn-rate. The turn-rate is part of the target kinematics, and thus the extension time evolution is not independent of the kinematical state. In this paper we relax the assumption (12) to be able to model transformations of the extension that are dependent on the kinematic state. 
Regarding the time evolution of the kinematic state, we adopt the same assumption as [8],

$$
p\left(\mathbf{x}_{k+1} \mid X_{k+1}, \mathbf{x}_{k}\right)=p\left(\mathbf{x}_{k+1} \mid \mathbf{x}_{k}\right) .
$$

This assumption neglects factors such as wind resistance, which can be modeled as a function of the target size. However, constructing a model of such phenomena is not easy in the general case, and could lead to overly complicated mathematics. Further, the uncertainty that is neglected in this approximation is, to a certain degree, captured by the process noise. This gives the following transition density,

$$
p\left(\xi_{k+1} \mid \xi_{k}\right) \triangleq p\left(\mathbf{x}_{k+1} \mid \mathbf{x}_{k}\right) p\left(X_{k+1} \mid \xi_{k}\right) .
$$

The integral (10), with posterior distribution (5b) and transition density (14), is

$$
\begin{aligned}
& \iint p\left(\mathbf{x}_{k+1} \mid \mathbf{x}_{k}\right) p\left(X_{k+1} \mid \xi_{k}\right) \\
& \times p\left(\mathbf{x}_{k} \mid \mathbf{Z}^{k}\right) p\left(X_{k} \mid \mathbf{Z}^{k}\right) \mathrm{d} \mathbf{x}_{k} \mathrm{~d} X_{k} \\
= & \int p\left(\mathbf{x}_{k+1} \mid \mathbf{x}_{k}\right) \int p\left(X_{k+1} \mid \xi_{k}\right) p\left(X_{k} \mid \mathbf{Z}^{k}\right) \mathrm{d} X_{k} \\
& \quad \times p\left(\mathbf{x}_{k} \mid \mathbf{Z}^{k}\right) \mathrm{d} \mathbf{x}_{k} \\
= & \int p\left(\mathbf{x}_{k+1} \mid \mathbf{x}_{k}\right) p\left(X_{k+1} \mid \mathbf{x}_{k}, \mathbf{Z}^{k}\right) p\left(\mathbf{x}_{k} \mid \mathbf{Z}^{k}\right) \mathrm{d} \mathbf{x}_{k} \\
= & p\left(\mathbf{x}_{k+1}, X_{k+1} \mid \mathbf{Z}^{k}\right) .
\end{aligned}
$$

As noted above, we want the predicted pdf to be of the same functional form as the posterior pdf (5b), however in general it does not hold that

$$
p\left(\mathbf{x}_{k+1}, X_{k+1} \mid \mathbf{Z}^{k}\right)=p\left(\mathbf{x}_{k+1} \mid \mathbf{Z}^{k}\right) p\left(X_{k+1} \mid \mathbf{Z}^{k}\right) .
$$

Therefore, to simplify further discussion and to obtain practical tractability we solve

$$
\begin{aligned}
& \int p\left(\mathbf{x}_{k+1} \mid \mathbf{x}_{k}\right) p\left(\mathbf{x}_{k} \mid \mathbf{Z}^{k}\right) \mathrm{d} \mathbf{x}_{k} \\
& \times \int p\left(X_{k+1} \mid \mathbf{x}_{k}, \mathbf{Z}^{k}\right) p\left(\mathbf{x}_{k} \mid \mathbf{Z}^{k}\right) \mathrm{d} \mathbf{x}_{k}
\end{aligned}
$$

instead of solving (15c). Note that in [7], [10] an assumption ${ }^{2}$ is made in order to simplify discussion and obtain tractability of the kinematic state prediction. This is equivalent to solving

$$
\begin{aligned}
p\left(\mathbf{x}_{k+1} \mid \mathbf{Z}^{k}\right) & =\int p\left(\mathbf{x}_{k+1} \mid \mathbf{x}_{k}\right) p\left(\mathbf{x}_{k} \mid \mathbf{Z}^{k}\right) \mathrm{d} \mathbf{x}_{k}, \\
p\left(X_{k+1} \mid \mathbf{Z}^{k}\right) & =\int p\left(X_{k+1} \mid \xi_{k}\right) p\left(\xi_{k} \mid \mathbf{Z}^{k}\right) \mathrm{d} \xi_{k},
\end{aligned}
$$

instead of solving (10), and gives a predicted pdf that is of the same functional form as the posterior pdf.

The prediction (18) does neglect some dependence between the kinematic state and the extension state, just like the measurement update (see [8]) neglects some dependence. However, the posterior kinematic state is always used to predict the extension state, which provides for interdependency between the two estimates, and as noted above by quoting from [8], the measurement update also provides for further

\footnotetext{
${ }^{2}$ The assumption in [7], [10] is different than that in (17), see [7] for comments and justification.
}

interdependency. It will be shown in the results section that good estimation performance can be achieved also under this independence assumption.

For the kinematical state, the transition density is modelled as

$$
p\left(\mathbf{x}_{k+1} \mid \mathbf{x}_{k}\right) \triangleq \mathcal{N}\left(\mathbf{x}_{k+1} ; f\left(\mathbf{x}_{k}\right), Q_{k+1}\right),
$$

where $f(\cdot): \mathbb{R}^{n_{x}} \rightarrow \mathbb{R}^{n_{x}}$ is a state transition function, and $Q_{k+1}$ is the process noise covariance for the kinematic state. The function $f(\cdot)$ is generally nonlinear, see [13] for a thorough overview of state transition functions.

Generalizing the Wishart transition densities of [7], [10], described in (8) and (9), the extension transition density is modeled as

$$
p\left(X_{k+1} \mid \xi_{k}\right) \triangleq \mathcal{W}_{d}\left(X_{k+1} ; n_{k+1}, \frac{M_{\mathbf{x}_{k}} X_{k} M_{\mathbf{x}_{k}}^{\mathrm{T}}}{n_{k+1}}\right),
$$

where $n_{k+1}>d-1$ is a scalar design parameter, and the matrix transformation $M_{\mathbf{x}_{k}} \triangleq M\left(\mathbf{x}_{k}\right): \mathbb{R}^{n_{x}} \rightarrow \mathbb{R}^{d \times d}$ is a non-singular matrix valued function of the kinematic state. The extension state's time evolution is modeled as being dependent on the kinematical state via a matrix transformation. The main motivation for this specific form is the modeling of rotation of extended targets. However, in general the function $M_{\mathbf{x}_{k}}$ can be selected arbitrarily, as long as the output is a non-singular $d \times d$ matrix. In terms of, e.g., group target tracking, the extension can grow or shrink over time, corresponding to $M_{\mathbf{x}_{k}}$ being a scaling of the extension.

The scalar design parameter $n_{k+1}$ in (20) is analogous to the noise covariance $Q_{k+1}$ in (19), i.e. it governs the extension state process noise. Let $\mathbb{M}_{k}=M_{\mathbf{x}_{k}} X_{k} M_{\mathbf{x}_{k}}^{\mathrm{T}}$ and let $X^{[i j]}$ denote the $(i, j)$ th element of the matrix $X$. By [11, Theorem 3.3.15] the expected value and variance of the $(i, j)$ th element of $X_{k+1} \mid \mathbf{x}_{k}, X_{k}$ are

$$
\begin{aligned}
\mathrm{E}\left[X_{k+1}^{[i j]} \mid \mathbf{x}_{k}, X_{k}\right] & =\mathbb{M}_{k}^{[i j]}, \\
\operatorname{Var}\left(X_{k+1}^{[i j]} \mid \mathbf{x}_{k}, X_{k}\right) & =\frac{\left(\mathbb{M}_{k}^{[i j]}\right)^{2}}{n_{k+1}}+\frac{\mathbb{M}_{k}^{[i i]} \mathbb{M}_{k}^{[j j]}}{n_{k+1}},
\end{aligned}
$$

i.e., given $\mathbf{x}_{k}$ and $X_{k}$ the variance decreases with increasing $n_{k+1}$. It can thus be said that a higher $n_{k+1}$ implies less process noise for the extension state. Thus, the shorter the prediction time interval $T$ is, the larger $n_{k+1}$ should be, and in the $\operatorname{limit}_{T \rightarrow 0} n_{k+1}=\infty$ should hold. One way to model $n_{k+1}$ as a function of prediction time is $n_{k+1}=n e^{-T / \tau}$ [7], [9], with two scalar parameters $n$ and $\tau$. We elaborate further on $n_{k+1}$ after we derive the main result of the paper.

The problem considered in this work is to, given a posterior density (5c) and the transition densities (14), (19), (20), obtain a solution to (18), where the predicted density $p\left(\xi_{k+1} \mid \mathbf{Z}^{k}\right)$ is of the same functional form as $(5 \mathrm{c})$, i.e.

$$
\begin{aligned}
p\left(\xi_{k+1} \mid \mathbf{Z}^{k}\right)= & p\left(\mathbf{x}_{k+1} \mid \mathbf{Z}^{k}\right) p\left(X_{k+1} \mid \mathbf{Z}^{k}\right) \\
= & \mathcal{N}\left(\mathbf{x}_{k+1} ; m_{k+1 \mid k}, P_{k+1 \mid k}\right) \\
& \times \mathcal{I} \mathcal{W}_{d}\left(X_{k+1} ; \nu_{k+1 \mid k}, V_{k+1 \mid k}\right) .
\end{aligned}
$$




\section{Preliminaries}

In this section we first give some known results, and then give three theorems, which are all needed in our derivation of a prediction update. For the pdf approximations below, the true densities are approximated by the minimization of the Kullback-Leibler divergence (KL-div) [14]. The KL-div is defined for two pdfs $p(x)$ and $q(x)$ as

$$
\mathrm{KL}(p(x) \| q(x))=\int p(x) \log (p(x) / q(x)) \mathrm{d} x .
$$

Note that, when it comes to approximating distributions in a maximum likelihood sense, the KL-div is considered the optimal difference measure [15]-[17].

\section{A. Known results}

Let $p(X)=\mathcal{W}_{d}(X ; v, V)$, and let $M$ be any non-singular $d \times d$ matrix. The random matrix $M X M$ is distributed as [11, Theorem 3.3.1]

$$
p(M X M)=\mathcal{W}_{d}(M X M ; v, M V M) .
$$

Let $p(X \mid V)=\mathcal{W}_{d}(X ; n, V)$ and let $p(V)=$ $\mathcal{I W}_{d}(V ; \bar{v}, \bar{V})$. The marginal for $X$ is [11, Problem 5.33]

$$
p(X)=\mathcal{G B}_{d}^{I I}\left(X ; \frac{n}{2}, \frac{\bar{v}-d-1}{2}, \bar{V}, \mathbf{0}_{d}\right) .
$$

B. Approximating a $\mathcal{G B}_{d}^{I I}$-distribution with an $\mathcal{I W}_{d^{-}}$ distribution

Theorem 1: Let $p(X)=\mathcal{G B}_{d}^{I I}\left(X ; a, b, \Omega, \mathbf{0}_{d}\right)$, and let $q(X)=\mathcal{I W}_{d}(X ; v, V)$ be the minimizer of the KullbackLeibler (KL) divergence between $p(X)$ and $q(X)$ among all $\mathcal{I W}_{d}$-distributions, i.e.

$$
q(X) \triangleq \underset{q(\cdot)=\mathcal{I} \mathcal{W}_{d}(\cdot)}{\arg \operatorname{KL}}(p(X) \| q(X)) .
$$

Then $V$ is given as

$$
V=\frac{(v-d-1)(2 a-d-1)}{2 b} \Omega,
$$

and $v$ is the solution to the equation

$$
\begin{aligned}
& \sum_{i=1}^{d}\left[\psi_{0}\left(\frac{2 a+1-i}{2}\right)-\psi_{0}\left(\frac{2 b+1-i}{2}\right)\right. \\
& \left.+\psi_{0}\left(\frac{v-d-i}{2}\right)\right]-d \log \left(\frac{(v-d-1)(2 a-d-1)}{4 b}\right)=0,
\end{aligned}
$$

where $\psi_{0}(\cdot)$ is the digamma function (a.k.a. the polygamma function of order 0 ).

Proof: Given in a technical report [18, Online] due to space considerations.

The equations for $V(27)$ and $v(28)$ in Theorem 1 correspond to matching the expected value of $X^{-1}$ and $\log |X|$,

$$
\begin{aligned}
\mathrm{E}_{q}\left[X^{-1}\right] & =\mathrm{E}_{p}\left[X^{-1}\right], \\
\mathrm{E}_{q}[\log |X|] & =\mathrm{E}_{p}[\log |X|] .
\end{aligned}
$$

Notice that in Theorem 1, substituting a value for $v$ into (27) gives the analytical solution for $V$. The parameter $v$ can be found by applying a numerical root-finding algorithm to (28), see e.g. [19, Section 5.1]. Examples include Newton-Raphson or modified Newton algorithms, see e.g. [19, Section 5.4], for more alternatives see e.g. [19, Chapter 5]. In the following corollary, we supply an alternative to root-finding to obtain a value for $v$.

Corollary 1: A closed form solution for $v$ can be obtained using only (27) together with matching the first order moments. The expected values of the densities $p(\cdot)$ and $q(\cdot)$ are [11, Theorems 5.3.20, 3.4.3]

$$
\begin{aligned}
& \mathrm{E}_{p}[X]=\frac{2 a}{2 b-d-1} \Omega, \\
& \mathrm{E}_{q}[X]=\frac{V}{v-2 d-2}=\frac{v-d-1}{v-2 d-2} \frac{2 a-d-1}{2 b} \Omega .
\end{aligned}
$$

Equating the expected values and solving for $v$ gives

$$
v=(d+1) \frac{\frac{2 a-d-1}{2 b}-2 \frac{2 a}{2 b-d-1}}{\frac{2 a-d-1}{2 b}-\frac{2 a}{2 b-d-1}} .
$$

Matching the expected values, as in Corollary 1, can be seen as an approximation of matching the expected values of the $\log$ determinant (29b). Indeed, with numerical simulations one can show that the $v$ given by (31) is approximately equal to the solution of (28), the difference is typically on the order of one tenth of a degree of freedom.

References [7], [10], [11] contain discussions about using moment matching to approximate a $\mathcal{G B}_{d}^{I I}$-distribution with a $\mathcal{I W}_{d}$-distribution. Theorem 1 defines an approximation by minimising the KL divergence, which results in matching the expected values (29). The KL criterion is well-known in the literature for its moment-matching characteristics, see e.g. [20], [21].

\section{Approximating the density of $\mathbb{V}_{\mathrm{x}}$ with a $\mathcal{W}_{d}$-distribution}

Theorem 2: Let $\mathrm{x}$ be Gaussian distributed with mean $m$ and covariance $P$, and let $\mathbb{V}_{\mathbf{x}} \triangleq \mathbb{V}(\mathbf{x}) \in \mathbb{S}_{++}^{n_{x}}$ be a matrix valued function of $\mathbf{x}$. Let $p\left(\mathbb{V}_{\mathbf{x}}\right)$ be the density of $\mathbb{V}_{\mathbf{x}}$ induced by the random variable $\mathbf{x}$, and let $q\left(\mathbb{V}_{\mathbf{x}}\right)=\mathcal{W}_{d}\left(\mathbb{V}_{\mathbf{x}} ; s, S\right)$ be the minimizer of the KL-divergence between $p\left(\mathbb{V}_{\mathbf{x}}\right)$ and $q\left(\mathbb{V}_{\mathbf{x}}\right)$ among all $\mathcal{W}$-distributions, i.e.

$$
q\left(\mathbb{V}_{\mathbf{x}}\right) \triangleq \underset{q(\cdot)=\mathcal{W}(\cdot)}{\arg \min } \operatorname{KL}\left(p\left(\mathbb{V}_{\mathbf{x}}\right) \| q\left(\mathbb{V}_{\mathbf{x}}\right)\right) .
$$

Then $S$ is given as

$$
S=\frac{1}{s} \mathbb{C}_{I I}
$$

and $s$ is the solution to the equation

$$
d \log \left(\frac{s}{2}\right)-\sum_{i=1}^{d} \psi_{0}\left(\frac{s-i+1}{2}\right)+\mathbb{C}_{I}-\log \left|\mathbb{C}_{I I}\right|=0
$$

where $\mathbb{C}_{I} \triangleq \mathrm{E}\left[\log \left|\mathbb{V}_{\mathbf{x}}\right|\right]$ and $\mathbb{C}_{I I} \triangleq \mathrm{E}\left[\mathbb{V}_{\mathbf{x}}\right]$.

Proof: Given in a technical report [18, Online] due to space considerations. 
Corollary 2: $\mathbb{C}_{I}$ and $\mathbb{C}_{I I}$ can be calculated using a Taylor series expansion of $\mathbb{V}_{\mathbf{x}}$ around $\mathbf{x}=m$. A third order expansion yields

$$
\begin{aligned}
& \mathbb{C}_{I} \approx \log \left|\mathbb{V}_{m}\right|+\left.\sum_{i=1}^{n_{x}} \sum_{j=1}^{n_{x}} \frac{d^{2} \log \left|\mathbb{V}_{\mathbf{x}}\right|}{d \mathbf{x}_{j} d \mathbf{x}_{i}}\right|_{\mathbf{x}=m} P_{i j}, \\
& \mathbb{C}_{I I} \approx \mathbb{V}_{m}+\left.\sum_{i=1}^{n_{x}} \sum_{j=1}^{n_{x}} \frac{d^{2} \mathbb{V}_{\mathbf{x}}}{d \mathbf{x}_{j} d \mathbf{x}_{i}}\right|_{x=m} P_{i j} .
\end{aligned}
$$

In (35) the $i$ :th element of the vector $\mathbf{x}$ and the $i, j$ :th element of the matrix $P$ are $\mathbf{x}_{i}$ and $P_{i j}$, respectively. Moreover, the matrix $\mathbb{V}_{m}$ is the function $\mathbb{V}_{\mathbf{x}}$ evaluated at the mean $m$ of the random variable $\mathbf{x}$.

The equations for $S$ (33) and $s$ (34) in Theorem 2 correspond to matching the expected values of $\mathbb{V}_{\mathbf{x}}$ and $\log \left|\mathbb{V}_{\mathbf{x}}\right|$,

$$
\begin{aligned}
\mathrm{E}_{q}\left[\mathbb{V}_{\mathbf{x}}\right] & =\mathrm{E}_{p}\left[\mathbb{V}_{\mathbf{x}}\right], \\
\mathrm{E}_{q}\left[\log \left|\mathbb{V}_{\mathbf{x}}\right|\right] & =\mathrm{E}_{p}\left[\log \left|\mathbb{V}_{\mathbf{x}}\right|\right] .
\end{aligned}
$$

Similarly to (28), numerical root-finding can be used to calculate a solution to (34). Note that using a moment matching approach similar to Corollary 1 to find a value for $s$ is not advisable, since this would lead to further approximations (because the true distribution $p\left(\mathbb{V}_{\mathbf{x}}\right)$ is unknown), and would possibly require a more complicated numerical solution. For $s>d-1$ and any $S \in \mathbb{S}_{++}^{d}$ there is a unique root to (34), see [18] for a proof.

\section{Marginalising $\mathcal{I W}_{d}(X \mid V) \mathcal{W}(V)$ over $V$}

This result is similar to the property stated in (25) and in [11, Problem 5.33].

Theorem 3: Let $p(X \mid V)=\mathcal{I}_{d}(X ; v, V / \gamma)$ and let $p(V)=\mathcal{W}_{d}(V ; s, S)$. The marginal for $X$ is

$$
p(X)=\mathcal{G B}_{d}^{I I}\left(X ; \frac{s}{2}, \frac{v-d-1}{2}, \frac{S}{\gamma}, \mathbf{0}_{d}\right) .
$$

Proof: Given in a technical report [18, Online] due to space considerations.

\section{A NEW PREDICTION UPDATE FOR THE EXTENSION}

In this section we present the new approach to prediction, first for the kinematical state in Section V-A and the for the extension state in Section V-B.

\section{A. Predicting the kinematical state}

For the kinematical state we have

$$
\begin{aligned}
p\left(\mathbf{x}_{k+1} \mid \mathbf{Z}^{k}\right)=\int \mathcal{N} & \left(\mathbf{x}_{k+1} ; f\left(\mathbf{x}_{k}\right), Q\right) \\
& \times \mathcal{N}\left(\mathbf{x}_{k} ; m_{k \mid k}, P_{k \mid k}\right) \mathrm{d} \mathbf{x}_{k},
\end{aligned}
$$

In case $f\left(\mathbf{x}_{k}\right)$ is a linear function, the solution to the integral (38) is given by the Kalman filter prediction [12]. In general $f\left(\mathbf{x}_{k}\right)$ is non-linear, in which case it is straightforward to solve the integral (38) approximately. Using the extended
Kalman filter prediction formulas, see e.g. [22], the predicted mean $m_{k+1 \mid k}$ and covariance $P_{k+1 \mid k}$ are

$$
m_{k+1 \mid k}=f\left(m_{k \mid k}\right), \quad P_{k+1 \mid k}=F_{k \mid k} P_{k \mid k} F_{k \mid k}^{\mathrm{T}}+Q
$$

where $\left.F_{k \mid k} \triangleq \nabla_{\mathbf{x}} f(\mathbf{x})\right|_{\mathbf{x}=m_{k \mid k}}$ is the gradient of $f(\cdot)$ evaluated at the mean $m_{k \mid k}$.

\section{B. Predicting the extension state}

For the extension state we have

$$
\begin{aligned}
& p\left(X_{k+1} \mid \mathbf{Z}^{k}\right)=\iint p\left(X_{k+1} \mid \mathbf{x}_{k}, X_{k}\right) p\left(\mathbf{x}_{k}, X_{k} \mid \mathbf{Z}^{k}\right) \mathrm{d} \mathbf{x}_{k} \mathrm{~d} X_{k} \\
&=\iint \mathcal{W}_{d}\left(X_{k+1} ; n_{k+1}, \frac{M_{\mathbf{x}_{k}} X_{k} M_{\mathbf{x}_{k}}^{\mathrm{T}}}{n_{k+1}}\right) \\
& \times \mathcal{N}\left(\mathbf{x}_{k} ; m_{k \mid k}, P_{k \mid k}\right) \\
& \times \mathcal{I} \mathcal{W}_{d}\left(X_{k} ; \nu_{k \mid k}, V_{k \mid k}\right) \mathrm{d} \mathbf{x}_{k} \mathrm{~d} X_{k} .
\end{aligned}
$$

Using the properties given in (24) and (25), the integral (40b) becomes

$$
\begin{aligned}
& p\left(X_{k+1} \mid \mathbf{Z}^{k}\right) \\
=\int & \mathcal{G} \mathcal{B}_{d}^{I I}\left(X_{k+1} ; \frac{n_{k+1}}{2}, \frac{\nu_{k \mid k}-d-1}{2}, \frac{M_{\mathbf{x}_{k}} V_{k \mid k} M_{\mathbf{x}_{k}}^{\mathrm{T}}}{n_{k+1}}, \mathbf{0}_{d}\right) \\
\quad & \mathcal{N}\left(\mathbf{x}_{k} ; m_{k \mid k}, P_{k \mid k}\right) \mathrm{d} \mathbf{x}_{k} .
\end{aligned}
$$

Unfortunately, the integral (41) has no analytical solution, it has to be solved using approximations.

In what follows, we first show how (7) can be heuristically modified to allow for transformations of the extension, and then the prediction method from [10] is briefly described. Lastly the main result of the paper is given: a new prediction update for the extension state.

1) Heuristic modification of (7): Note first that the prediction (7) corresponds to the case $M_{\mathbf{x}_{k}}=\mathbf{I}_{d}$. The prediction (7) is hereafter called method 1 (M1).

Including a non-identity transformation matrix $M(\cdot)$ in the prediction process can be done heuristically, e.g. by replacing (7b) with

$$
V_{k+1 \mid k}=\frac{\nu_{k+1 \mid k}-2 d-2}{\nu_{k \mid k}-2 d-2} M_{m_{k \mid k}} V_{k \mid k} M_{m_{k \mid k}}^{\mathrm{T}} .
$$

This prediction for the extension evaluates $M_{\mathbf{x}_{k}}$ at the last estimated kinematic state $m_{k \mid k}$, and can thus capture e.g. rotations. However, it neglects the kinematic state uncertainty $P_{k \mid k}$ completely. The prediction given by (7a) and (42) is hereafter called method 2 (M2).

2) Prediction method from [10]: An alternative to (42) is to replace $M_{\mathbf{x}_{k}}$ by $M_{m_{k \mid k}}$ in (41). In this case the integral (41) has an analytical solution, and Theorem 1 can then be used to approximate the the $\mathcal{G} \mathcal{B}_{d}^{I I}$-density as an $\mathcal{I W}_{d}$-distribution. A similar approach is taken in [10], and the extension transition density used in [10] was given in (9). The matrix $A_{k}$ in (9) is a parameter, and is not dependent on the kinematical state. In [10] the authors use a type of moment matching to approximate the density, instead of minimization of the KL-div. 
The prediction method from [10] is hereafter called method 3 (M3).

Note that if $A_{k}=M_{m_{k \mid k}} / \sqrt{\delta_{k}}$, and if $\tau$ and $\delta_{k}$ are chosen correctly, M2 is equivalent to M3. In Section VI-A we show how $\tau$ can be chosen for this equivalence to hold. The transition density (9) is used in a multiple model framework in [10], with $m$ different modes with corresponding parameters $\delta_{k}^{(m)}$ and $A_{k}^{(m)}$. The extension modes correspond to, e.g., no rotation, rotation $\theta \mathrm{rad}$, and rotation $-\theta \mathrm{rad}$. In the results section it will be clear from context if it is the single mode, or multiple mode, version of M3 that is referred to.

3) New prediction for the extension state: Using Theorem 1 , the $\mathcal{G} \mathcal{B}_{d}^{I I}$-distribution in (41) can be approximated as an $\mathcal{I} \mathcal{W}_{d}$-distribution,

$$
\begin{aligned}
p\left(X_{k+1} \mid \mathbf{Z}^{k}\right) \approx \int & \mathcal{I} \mathcal{W}_{d}\left(X_{k+1} ; v_{k \mid k}, \frac{M_{\mathbf{x}_{k}} V_{k \mid k} M_{\mathbf{x}_{k}}^{\mathrm{T}}}{\gamma_{k \mid k}}\right) \\
& \times \mathcal{N}\left(\mathbf{x}_{k} ; m_{k \mid k}, P_{k \mid k}\right) \mathrm{d} \mathbf{x}_{k},
\end{aligned}
$$

where $v_{k \mid k}$ is calculated using Corollary 1 by setting $a=$ $\frac{n_{k+1}}{2}, b=\frac{\nu_{k \mid k}-d-1}{2}$, and $\gamma_{k \mid k} \triangleq \frac{2 b n_{k+1}}{\left(v_{k \mid k}-d-1\right)(2 a-d-1)}$. Using the variable substitution $\mathbb{V}_{\mathbf{x}_{k}} \triangleq M_{\mathbf{x}_{k}} V_{k \mid k} M_{\mathbf{x}_{k}}^{\mathrm{T}}$, we obtain

$$
\begin{aligned}
p\left(X_{k+1} \mid \mathbf{Z}^{k}\right) \approx & \mathcal{I} \mathcal{W}_{d}\left(X_{k+1} ; v_{k \mid k}, \frac{M_{\mathbf{x}_{k}} V_{k \mid k} M_{\mathbf{x}_{k}}^{\mathrm{T}}}{\gamma_{k \mid k}}\right) \\
& \times \mathcal{N}\left(\mathbf{x}_{k} ; m_{k \mid k}, P_{k \mid k}\right) \mathrm{d} \mathbf{x}_{k} \\
= & \int \mathcal{I} \mathcal{W}_{d}\left(X_{k+1} ; v_{k \mid k}, \frac{\mathbb{V}_{\mathbf{x}_{k}}}{\gamma_{k \mid k}}\right) p\left(\mathbb{V}_{\mathbf{x}_{k}}\right) \mathrm{d} \mathbb{V}_{\mathbf{x}_{k}} .
\end{aligned}
$$

In (44a) the $\mathcal{I} \mathcal{W}_{d}$ density depends on $\mathbf{x}_{k}$ only through $M_{\mathbf{x}_{k}} V_{k \mid k} M_{\mathbf{x}_{k}}^{\mathrm{T}}$, and the second equality then follows as a result of the variable substitution and standard probability theory for variable substitutions, see e.g. [23, Theorem 2.1]. Note that $\mathbb{V}_{\mathbf{x}_{k}}$ is a $d \times d$ random matrix, and $p\left(\mathbb{V}_{\mathbf{x}_{k}}\right)$ is a matrix variate density. Because exact calculation of $p\left(\mathbb{V}_{\mathbf{x}_{k}}\right)$ is prohibitively difficult, we use Theorem 2 to approximate $p\left(\mathbb{V}_{\mathbf{x}_{k}}\right)$ by a Wishart distribution. ${ }^{3}$ This gives

$$
\begin{aligned}
p\left(X_{k+1} \mid \mathbf{Z}^{k}\right) \approx \int & \mathcal{I} \mathcal{W}_{d}\left(X_{k+1} ; v_{k \mid k}, \frac{\mathbb{V}_{\mathbf{x}_{k}}}{\gamma_{k \mid k}}\right) \\
& \times \mathcal{W}_{d}\left(\mathbb{V}_{\mathbf{x}_{k}} ; s_{k \mid k}, S_{k \mid k}\right) \mathrm{d} \mathbb{V}_{\mathbf{x}_{k}},
\end{aligned}
$$

where $s_{k \mid k}$ and $S_{k \mid k}$ are calculated by setting $m=m_{k \mid k}$ and $P=P_{k \mid k}$ in Theorem 2, and $\mathbb{C}_{I}$ and $\mathbb{C}_{I I}$ are computed using Corollary 2. Using Theorem 3 the marginal for $X_{k+1}$, which is the solution to the integral of (45), is given as

$$
p\left(X_{k+1} \mid \mathbf{Z}^{k}\right) \approx \mathcal{G B}_{d}^{I I}\left(X_{k+1} ; a_{k+1 \mid k}, b_{k+1 \mid k}, \Omega_{k+1 \mid k}, \mathbf{0}_{d}\right)
$$

where $a_{k+1 \mid k} \triangleq \frac{s_{k \mid k}}{2}, b_{k+1 \mid k} \triangleq \frac{v_{k \mid k}-d-1}{2}$ and $\Omega_{k+1 \mid k} \triangleq \frac{S_{k \mid k}}{\gamma_{k \mid k}}$. Finally, using Theorem 1 once again we obtain

$$
p\left(X_{k+1} \mid \mathbf{Z}^{k}\right) \approx \mathcal{I} \mathcal{W}_{d}\left(X_{k+1} ; \nu_{k+1 \mid k}, V_{k+1 \mid k}\right),
$$

\footnotetext{
${ }^{3}$ Note that Theorem 2 uses the parameters of $\mathcal{N}\left(\mathbf{x}_{k} ; m_{k \mid k}, P_{k \mid k}\right)$ in order to construct the Wishart approximation.
}

where the prediction updated parameters $\nu_{k+1 \mid k}$ and $V_{k+1 \mid k}$ are

$$
\begin{aligned}
\nu_{k+1 \mid k} & =(d+1) \frac{\frac{2 a_{k+1 \mid k}-d-1}{2 b_{k+1 \mid k}}-2 \frac{2 a_{k+1 \mid k}}{2 b_{k+1 \mid k}-d-1}}{\frac{2 a_{k+1 \mid k}-d-1}{2 b_{k+1 \mid k}}-\frac{2 a_{k+1 \mid k}}{2 b_{k+1 \mid k}-d-1}}, \\
V_{k+1 \mid k} & =\frac{\left(\nu_{k+1 \mid k}-d-1\right)\left(2 a_{k+1 \mid k}-d-1\right)}{2 b_{k+1 \mid k}} \Omega_{k+1 \mid k} .
\end{aligned}
$$

Hereafter this prediction update is called method 4 (M4). This method improves upon the prediction updates M2 and M3 by also considering the kinematic state uncertainty.

\section{Another look at the parameter $n_{k+1}$}

In this section we elaborate on the parameter $n_{k+1}$ in the extension state transition density. Under the assumption $M_{\mathbf{x}}=$ $\mathbf{I}_{d}$ we have

$p\left(X_{k+1} \mid \mathbf{Z}^{k}\right)=\mathcal{G B}_{d}^{I I}\left(X_{k+1} ; \frac{n_{k+1}}{2}, \frac{\nu_{k \mid k}-d-1}{2}, \frac{V_{k \mid k}}{n_{k+1}}, \mathbf{0}_{d}\right)$

and the expected value and variance of the $(i, j)$ th element $X_{k+1}^{[i j]}$ are

$$
\begin{aligned}
\mathrm{E}\left[X_{k+1}^{[i j]}\right] & =\mathrm{E}\left[X_{k}^{[i j]}\right], \\
\operatorname{Var}\left(X_{k+1}^{[i j]}\right) & =\underbrace{\left(1+\frac{\nu_{k \mid k}-2 d-2}{n_{k+1}}\right)}_{\triangleq \eta_{k+1}} \operatorname{Var}\left(X_{k}^{[i j]}\right) .
\end{aligned}
$$

We see that (50) corresponds to exponential forgetting prediction for the $(i, j)$ th element, see e.g. [24]. The forgetting factor is $0<\eta_{k+1}^{-1}<1$, and the effective window length is

$$
w_{e}=\frac{1}{1-\eta_{k+1}^{-1}}=1+\frac{n_{k+1}}{\nu_{k \mid k}-2 d-2}
$$

time steps. Using exponential forgetting prediction with window length $w_{e}$ approximately means that we only "trust" the information that was contained in the measurements from the last $w_{e}$ time steps. This gives us a hint as to how a specific value of $n_{k+1}$ could be set, either as a global constant, or dynamically for each individual target at each time step.

An alternative way to interpret $n_{k+1}$ starts with Corollary 1 . We can rewrite (31) to obtain

$$
\nu_{k+1 \mid k}=\nu_{k \mid k}-\underbrace{\frac{\left(\nu_{k \mid k}-2 d-2\right)\left(\nu_{k \mid k}-d-1\right)}{n_{k+1}+\nu_{k \mid k}-2 d-2}}_{N\left(\nu_{k \mid k}, n_{k+1}\right)},
$$

where $N(\cdot)$ is a scalar valued function of $\nu_{k \mid k}$ and $n_{k+1}$. This is analogous to the measurement update [8]

$$
\nu_{k+1 \mid k+1}=\nu_{k+1 \mid k}+N_{z, k+1},
$$

where $N_{z, k+1}$ is the number of measurements at time step $k+1$. Thus, we can view the prediction as "removing" degrees of freedom corresponding to $N\left(\nu_{k \mid k}, n_{k+1}\right)$ measurements. 


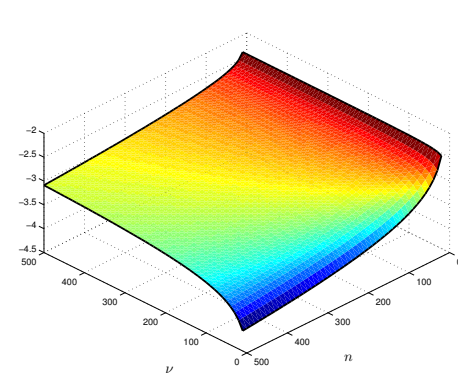

(a)

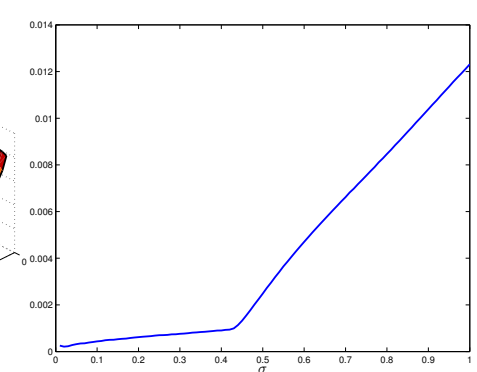

(b)

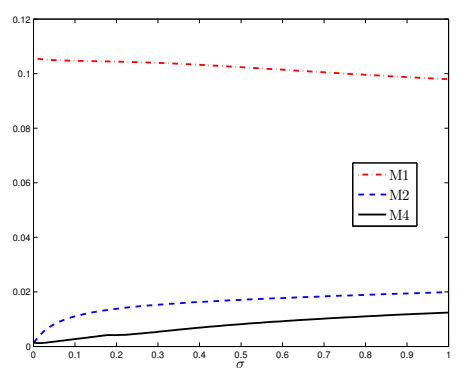

(c)

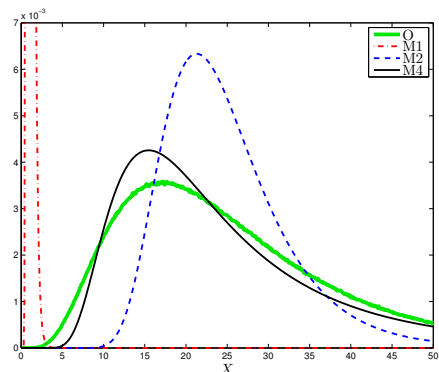

(d)

Fig. 1. Results for one dimensional extensions. (a) The log-distances $\log _{10}\left(\Delta_{\text {pdf }}(p(x), q(x))\right)$ when a $\mathcal{G} \mathcal{B}_{d}^{I I}$ is approximated as an $\mathcal{I} \mathcal{W}_{d}$. The approximation is least accurate when the parameter $n$ (cf. (20)) is small. (b). The distances $\Delta_{\text {pdf }}\left(p\left(\mathbb{V}_{\mathbf{x}}\right), \mathcal{W}\right)$ when $\mathbf{x}$ is normal distributed and the distribution over $\mathbb{V} \mathbf{x}$ is approximated as a $\mathcal{W}_{d}$. As expected, the accuracy of the approximation decreases when the uncertainty of $\mathbf{x}$ increases. (c) The distances $\Delta_{\text {pdf }}(\cdot, \cdot)$ between the empirical distribution and the three prediction methods for different values of $\sigma$. The same transformation function $M_{\mathbf{x}}$ and same values of $\sigma$ as were used in (b) are used here. (d) Comparison of the empirical distribution and the three different predicted distributions for $\sigma=0.3$, the legend refers to the empirical distribution $(\mathrm{O})$ and the three methods M1, M2 and M4. The suggested new prediction outperforms the other methods.

\section{Simulations}

This section presents results from simulations which compare the new prediction method M4 to the methods M1, M2, and M3. The main focus is on the prediction of the extension state.

In all simulations of M4, Corollary 1 is used to calculate $v_{k \mid k}$. For computing the quantity $s_{k \mid k}$, (34) is solved numerically using the iterative Halley's method [25]. This requires the digamma function to be computed, which is performed in MATLAB using the function psi $(\cdot)$. Simulations have shown that $s_{k \mid k}$ is computed, on average, in just 4 iterations. Note that the only part of M4 that requires a numerical solution is the calculation of $s_{k \mid k}$. All other quantities required are calculated using their respective closed form expressions.

In the following subsections, first a method to determine the parameter $\tau$ in M1 and M2 is given in Section VI-A, then a difference measure for pdfs is presented in Section VI-B. This is followed by simulation results for one dimensional extensions in Section VI-C, and for two dimensional extensions in Section VI-D.

\section{A. Determining $\tau$}

M1 and M2 contain the parameter $\tau$, which is a "time constant related to the agility with which the object may change its extension over time" [8]. Neither Koch [7] nor Feldmann et al [8] elaborate on how $\tau$ is best determined. To make as fair comparison as possible, here Theorem 1 is used to determine $\tau$. By Theorem 1, the following holds,

$$
\begin{aligned}
& \int \mathcal{I} \mathcal{W}_{d}\left(X_{+} ; n, \frac{X}{n}\right) \mathcal{W}_{d}(X ; v, V) d X \\
\approx & \mathcal{I} \mathcal{W}_{d}\left(X_{+} ; v_{+}, V_{+}\right) .
\end{aligned}
$$

By setting $v_{+}$equal to (7a), $\tau$ can be determined for any combination of $T, v$, and $n$. With this choice of $\tau$, all prediction methods yield the same result when $M_{\mathbf{x}}=\mathbf{I}_{d}$.

\section{B. Difference measure for probability density functions}

In order to measure the algorithms' prediction performances, a distance measure between two pdfs $p(x)$ and $q(x)$ is needed. Here the $L_{2}$-norm is used,

$$
\Delta_{\text {pdf }}(p(x), q(x)) \triangleq \int|p(x)-q(x)|^{2} d x .
$$

In order to calculate the integral numerically, a uniform discretization is made over the union of the supports of $p(x)$ and $q(x)$.

\section{Results in one dimension}

This section presents results for a one dimensional $(d=1)$ extension $X$. The kinematic state $\mathbf{x}_{k}$ is also selected as one dimensional, i.e. $n_{x}=d=1$. The transformation function $M\left(\mathbf{x}_{k}\right)$ is given as

$$
M\left(\mathbf{x}_{k}\right)=1+\mathbf{x}_{k}^{2}
$$

The integral in (55) is computed with a discretization over the interval $[0,1000]$ with a discretization interval of length 0.1 .

1) Accuracy of Theorem 1: The accuracy of Theorem 1, i.e. of the approximation

$$
\mathcal{G B}_{d}^{I I}\left(X_{+} ; \frac{n}{2}, \frac{\nu-d-1}{2}, \frac{V}{n}, \mathbf{0}_{d}\right) \approx \mathcal{I W}_{d}\left(X_{+} ; \nu_{+}, V_{+}\right)
$$

is evaluated for different values of the parameters $n$ and $\nu$ by computing $\Delta_{\text {pdf }}\left(\mathcal{G} \mathcal{B}_{d}^{I I}, \mathcal{I W}_{d}\right)$ for each combination of $n$ and $\nu$. The results, see Fig. 1a, show that the approximation is least accurate when $n$ is small. A small $n$ corresponds to a very short effective window $w_{e}$, see (51) and Section V-C.

2) Accuracy of Theorem 2: Let $\mathbb{V}_{\mathbf{x}}=M_{\mathbf{x}} V_{0} M_{\mathbf{x}}$, where $M_{\mathbf{x}}$ is given in (56) and $p(\mathbf{x})=\mathcal{N}(\mathbf{x} ; m, \sigma)$. The accuracy of Theorem 2, i.e. approximation of the pdf of $\mathbb{V}_{\mathbf{x}}$ with a Wishart distribution, is evaluated for different values of the parameter $\sigma$, when $m=2$ and $V_{0}=1$. For each $\sigma$, an empirical pdf $p\left(\mathbb{V}_{\mathbf{x}}\right)$ is computed using $10^{7}$ samples from $\mathcal{N}(\mathbf{x} ; m, \sigma)$. The results, see Fig. 1b, show that, as expected, the approximation becomes less accurate as $\sigma$ becomes larger. While the result in Fig. 1b is specific for the transformation (56), the observation that $\Delta_{\text {pdf }}\left(p\left(\mathbb{V}_{\mathbf{x}}\right), \mathcal{W}_{d}\right)$ increases with $\sigma$ can be expected to hold for other transformation functions as well. 
3) Accuracy of the new prediction: The following parameter settings are used for the distribution (5c),

$$
\begin{aligned}
& \nu_{k \mid k}=50, \quad V_{0}=1, \quad V_{k \mid k}=\left(\nu_{k \mid k}-2 d-2\right) V_{0}, \\
& n_{k+1}=50, \quad m_{k \mid k}=2, \quad P_{k \mid k}=\sigma_{k \mid k} \in[0.01,1] \text {. }
\end{aligned}
$$

For each $\sigma_{k \mid k}$ value, a total of $10^{7}$ samples were generated from (5c) and each sample is predicted by sampling from (20). The resulting empirical pdf (emp) over $X_{k+1}$ is compared to the pdfs obtained by M1, M2 and M4. Remember that when $\tau$ is computed as in Section VI-A and $A_{k}=M_{m_{k \mid k}} / \sqrt{\delta_{k}}$, M2 is equivalent to the single mode version of M3. For another choice of $A_{k}$, the error for M3 would be larger than for M2.

Fig. 1c shows $\Delta_{\text {pdf }}(\mathrm{emp}, \mathrm{M} i)$ for different values of the parameter $\sigma$. For all values of $\sigma$, M4 outperforms the other two methods. Fig. 1d shows the pdfs for the case $\sigma=0.3$. Again it is evident that M4 is the best approximation of the empirical distribution.

\section{Results in two dimensions}

This section presents results for a two dimensional $(d=$ 2) extension. A constant turn-rate (CT) model with polar velocity [13] is considered. The kinematic state $\mathbf{x}_{k}=$ $\left[\mathrm{x}_{k}, \mathrm{y}_{k}, \mathrm{v}_{k}, \phi_{k}, \omega_{k}\right]^{\mathrm{T}}$ contains the $\left(\mathrm{x}_{k}, \mathrm{y}_{k}\right)$-position in Cartesian coordinates, the speed $\mathrm{v}_{k}$, the heading $\phi_{k}$ and the turnrate $\omega_{k}$. With this kinematic state, it is intuitive to let the transformation function be a rotation matrix

$$
M\left(\mathbf{x}_{k}\right)=\left[\begin{array}{cc}
\cos \left(T \omega_{k}\right) & -\sin \left(T \omega_{k}\right) \\
\sin \left(T \omega_{k}\right) & \cos \left(T \omega_{k}\right)
\end{array}\right] .
$$

1) Performance evaluation: For single step prediction, the predicted expected values $\mathrm{E}\left[X_{k+1}\right]$ and covariances $\operatorname{Cov}\left(X_{k+1}\right)$ are compared. Because the covariance of the extension matrix is a $d^{2} \times d^{2}$ matrix [11, Definition 1.2.6], we are going to constrain ourselves to illustrate only the $d \times d$ covariance matrix of the diagonal entries of the predicted extension matrix.

For single and multiple maneuvering targets, the predicted root mean square errors (RMSE) are computed over $N_{s}$ Monte Carlo runs. The predicted kinematical state position RMSE, and the extension state RMSE, are computed as follows,

$$
\begin{aligned}
\operatorname{RMSE}_{k}^{\mathbf{x}} & =\left(\frac{1}{N_{s}} \sum_{i=1}^{N_{s}}\left(\hat{\mathrm{x}}_{k \mid k-1}^{(i)}-\mathrm{x}_{k}\right)^{2}+\left(\hat{\mathrm{y}}_{k \mid k-1}^{(i)}-\mathrm{y}_{k}\right)^{2}\right)^{\frac{1}{2}}, \\
\operatorname{RMSE}_{k}^{X} & =\left(\frac{1}{N_{s}} \sum_{i=1}^{N_{s}} \operatorname{Tr}\left(\left(\hat{X}_{k \mid k-1}^{(i)}-X_{k}\right)^{2}\right)\right)^{\frac{1}{2}}
\end{aligned}
$$

where $\mathrm{x}_{k}, \mathrm{y}_{k}$ and $X_{k}$ are the true position and extension, and $\hat{\mathrm{x}}_{k \mid k-1}^{(i)}, \hat{\mathrm{y}}_{k \mid k-1}^{(i)}$ and $\hat{X}_{k \mid k-1}^{(i)}$ are the predicted position and extension from the $i$ th Monte Carlo run.

2) Single step prediction: The following parameter settings are used for the distribution $(5 \mathrm{c})$.

$$
\begin{aligned}
\nu_{k \mid k} & =50, & V_{k \mid k} & =\left(\nu_{k \mid k}-2 d-2\right) V_{0}, \\
V_{0} & =\operatorname{diag}([5,2]), & n_{k+1} & =50, \\
\omega_{k \mid k} & =0 \text { or } 45[\mathrm{deg}], & P_{\omega} & =1 \text { or } 20[\mathrm{deg}],
\end{aligned}
$$

where $P_{\omega}$ is the standard deviation for $\omega_{k \mid k}$. For each of the four parameter combinations, a total of $10^{5}$ samples were generated, and each sample was then predicted by sampling from (20). The resulting sample mean $X_{k+1}^{\mathrm{O}}$ (of the extent matrix) and sample covariance $C_{k+1}^{\mathrm{O}}$ (of the diagonal elements of the extent matrix) are compared to the expected value and covariance given by M1, M2 and M4. Note again that M2 and single mode M3 gives equivalent results.

The results are shown in Figure 2. It is evident that M1 has the worst performance of all three methods, because it does not take the rotation of the extension into account. M2 performs identically to M4 when $P_{\omega}$ is small, however for larger $P_{\omega}$ the sample mean $X_{k+1}^{\mathrm{O}}$ is slightly distorted, which M2 does not capture, and M2's covariance is underestimated compared to $C_{k+1}^{\mathrm{O}} . \mathrm{M} 4$, in comparison, captures the distorted shape of the sample mean, and M4's covariance is not underestimated, rather it is slightly overestimated compared to $C_{k+1}^{\mathrm{O}}$. Overestimation of the covariance is in general seen as more benign than underestimation, which can cause instability. Moreover, the increase of the covariance over the correct one $C_{k+1}^{\mathrm{O}}$ can be interpreted as a compensation for the approximations made during the calculation. As a result, M4 outperforms M1 and M2 in terms of both the first and second order moments of the predicted pdf over $X_{k+1}$.

3) Single maneuvering target: Two single maneuvering target scenarios were simulated. In Figure $3 \mathrm{a}$ the target moves forward with constant velocity (CV) for 25 time steps, and then maneuvers with constant turn rate $(\mathrm{CT}) \omega_{k}=10 \mathrm{deg}$ per time step for 35 time steps. In Figure $3 \mathrm{~b}$ the target moves forward with constant velocity for 25 time steps, and then maneuvers with a variable turn rate $\omega_{k}$ for 50 time steps. The turn rate first increased from 0 to $20 \mathrm{deg}$ per time step, and then decreased to 0 deg per time step. The last five time steps is CV motion. In both scenarios, the target generated 10 measurements in each time step, and there were no clutter measurements.

For these two scenarios the multiple mode version of M3 was implemented, please refer to [10] for details. In the simulation study in [10], M3 is implemented with three extension evolution modes which correspond to (1) no change with small process noise, (2) rotation $\theta$ deg with large process noise, and (3) rotation $-\theta$ deg with large process noise, where $\theta$ is a manually set model parameter. In each mode, the kinematical state is predicted according to a CV model [10].

In the comparison the CT version of M4 outlined above is compared to M3 with three modes and M3 with five modes, denoted M3/3 and M3/5 for short. We have chosen to not compare M4 to the prediction method from [8], because [10] contains a simulation comparison between M3 with three modes and [8], and the results show that during manuevers M3 with three modes outperforms [8].

M3/3 was implemented with the same three modes as in [10]. M3/5 was implemented with extension evolution modes that correspond to (1) no change with small process noise, (2) rotation $2 \theta$ deg with large process noise, (3) rotation $\theta$ deg with large process noise, (4) rotation $-\theta$ deg with large process noise, and (5) rotation $-2 \theta$ deg with large process noise. Note that [10] also includes a model for the measurement update, however in this paper we study only the prediction update and 


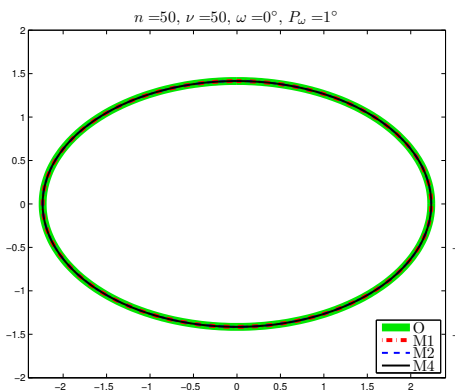

(a)

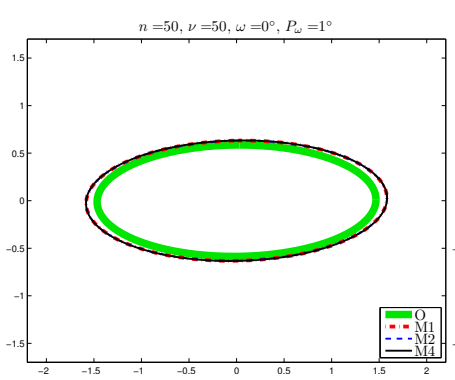

(e)

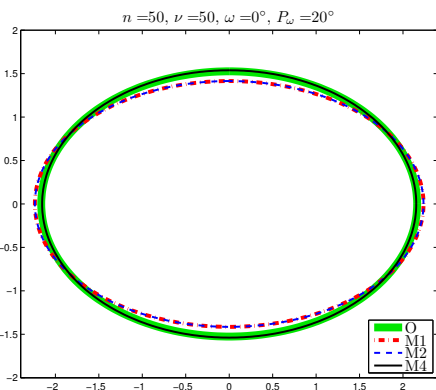

(b)

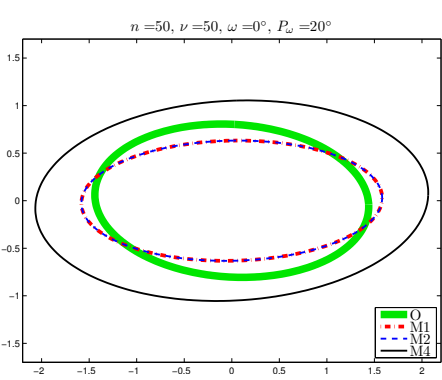

(f)

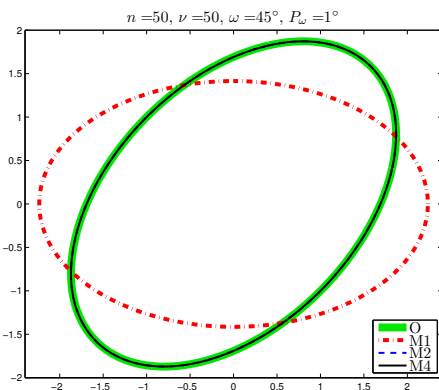

(c)

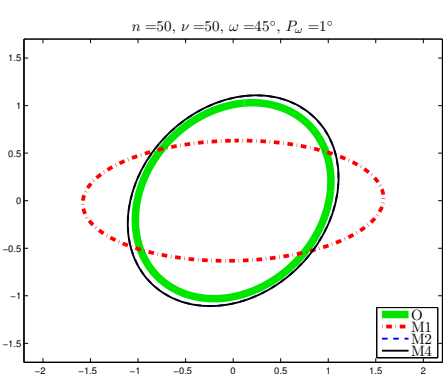

(g)

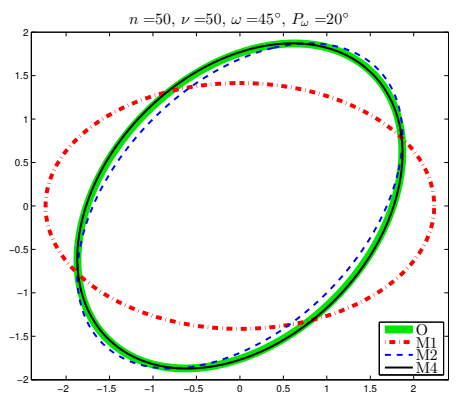

(d)

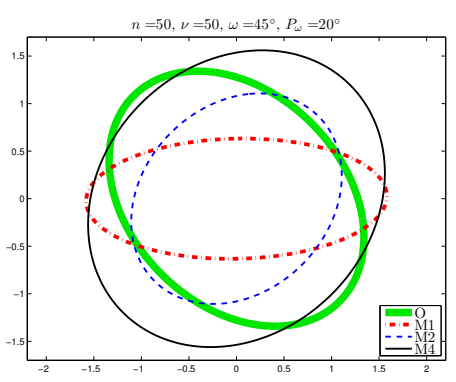

(h)

Fig. 2. Results for two dimensional extensions. The legend refers to the empirical distribution (O) and the three methods M1, M2 and M3. The top row shows a comparison of the expected value, the bottom row shows a comparison of the covariance matrices corresponding to the diagonal elements of the extension. Each column presents results for a different parameter setting.

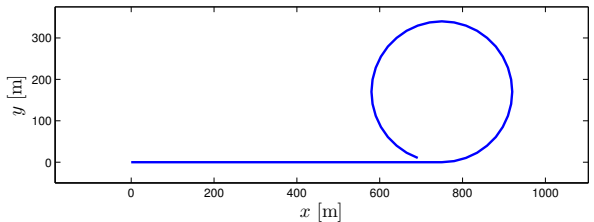

(a)

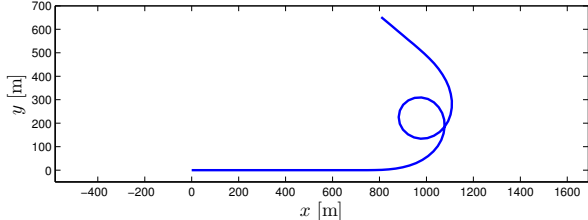

(b)

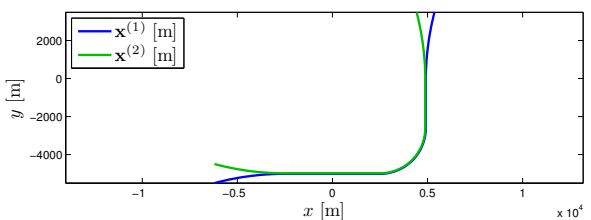

(c)

Fig. 3. True target tracks used in simulations. In (a) and (b) the target starts at the origin. In (c) the two targets start in the bottom left.

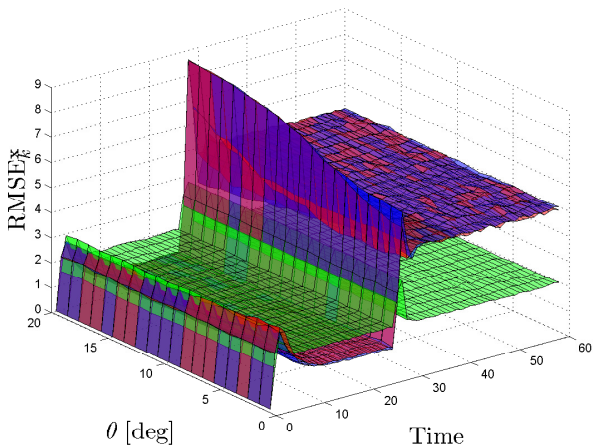

(a)

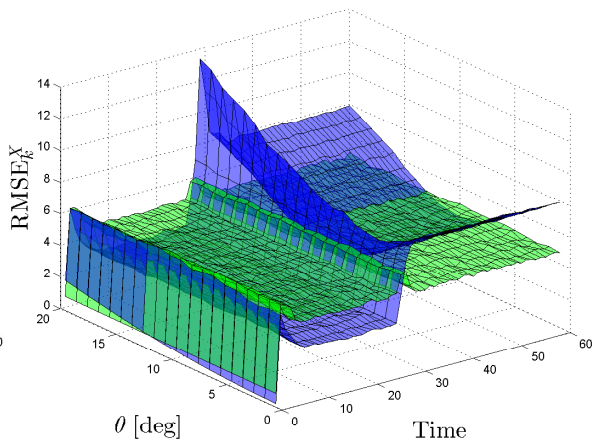

(b)

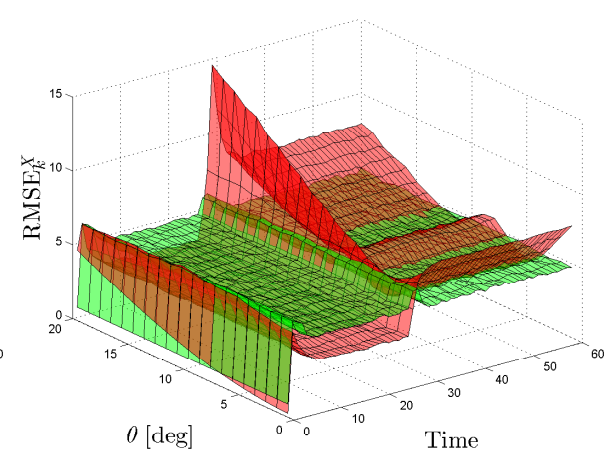

(c)

Fig. 4. Results for single target tracking for the true track in Fig. 3a. M3/3 in blue, M3/5 in red and M4 in green. (a): Position RMSE. (b) and (c): Extension RMSE, split into two figures for increased clarity. From time 25 to time 60 the true turn rate is 10 deg per time step. For M3/3 and M3/5, the position RMSE increases during the maneuver, but is independent of the rotation parameter $\theta$. However the extension RMSE increases with increasing parameter error. Note that for small parameter errors, M3/3 and M3/5 has lower extension RMSE than M4.

therefore use the standard measurement update [7], [8].

Both scenarios were simulated $N_{s}=1000$ times. To test M3's sensitivity to the parameter $\theta, \mathrm{M} 4$ is compared to M3 using $\theta \in[1,20] \mathrm{deg}$. Figures 4 and 5 show comparisons of the results from M3/3, M3/5 and M4 for the true target tracks in Figures $3 a$ and $3 b$, respectively. In the figures we see the following:

- M3/3 and M3/5 have lower errors when the target moves according to a $\mathrm{CV}$ model, because their $\mathrm{CV}$ model for the kinematics is better than M4's CT model for this type of 


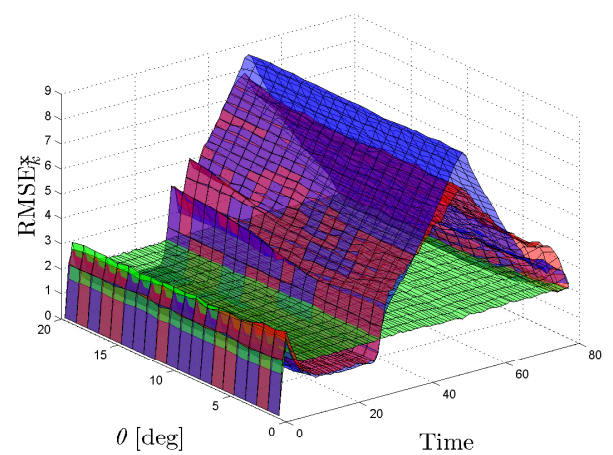

(a)

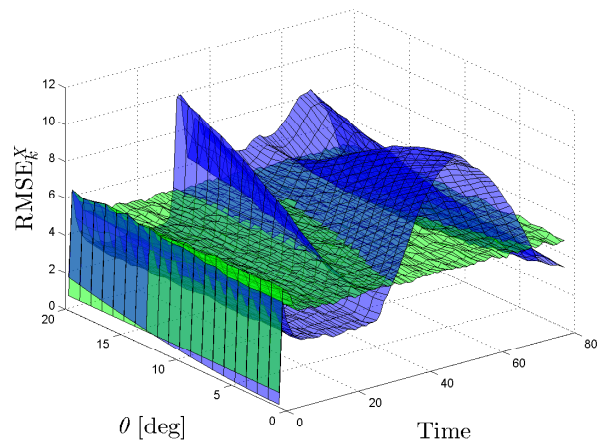

(b)

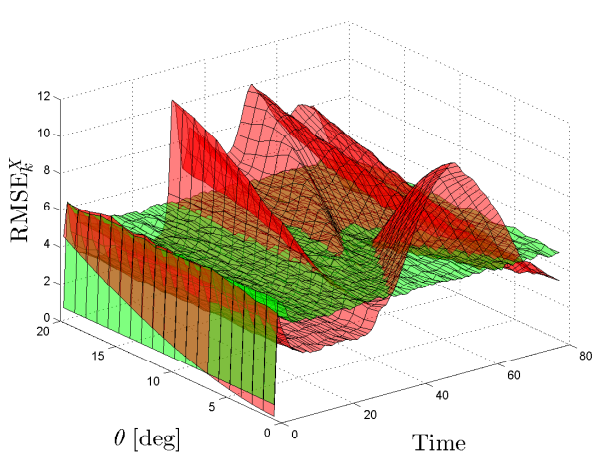

(c)

Fig. 5. Results for single target tracking for the true track in Fig. 3b. M3/3 in blue, M3/5 in red, and M4 in green . (a): Position RMSE. (b) and (c): Extension RMSE, split into two figures for increased clarity. From time 25 to time 75 the true turn rate goes from 0 deg to 20 deg per time step, and then back to 0 deg again. For M4 the position and extension RMSE are constant for the whole trajectory. For M3/3 and M3/5 the position RMSE is largest when the true turn rate is highest. During the maneuver, M4 has significantly smaller position and extension RMSEs than M3/3 and M3/5.

motion.

- During the manuevers, M4 has lower position error than M3/3 and M3/5 because the CT model is better than the $\mathrm{CV}$ model for this type of motion. The position errors for $\mathrm{M} 3 / 3$ and $\mathrm{M} 3 / 5$ increase with increasing turn-rate, see Figure 5a.

- During the CT maneuver M3/3 has lower extension error than M4 if $\theta \in(7 \mathrm{deg}, 13 \mathrm{deg})$ holds, see Figure 4b. For larger parameter errors, M4 is better because it estimates the turn-rate online. As the parameter error grows larger, M3/3's performance degrades more and more.

- During the CT maneuver M3/5 has lower extension error than M4 if $\theta \in(3.5 \mathrm{deg}, 6.5 \mathrm{deg})$ or $\theta \in$ (10.5 deg , $12.5 \mathrm{deg}$ ), see Figure 4c. The first interval corrsponds to $2 \theta \in(7 \mathrm{deg}, 13 \mathrm{deg})$, which is the same interval as for $\mathrm{M} 3 / 3$. Just as for $\mathrm{M} 3 / 3$, for larger parameter errors the performance of $\mathrm{M} 3 / 5$ degrades.

- In Figures 5b and 5c, M3/3 and M3/5 perform better than M4 during the time steps that correspond to a small parameter error. However, for the time steps where the parameter error is larger, M4 has significantly better performance than M3. Despite using two additional rotation modes, M3/5 does not have a clearly better performance than $\mathrm{M} 3 / 3$ for this type of maneuver.

In comparison, M3 and M4 are quite similar in that the extension transition density is a Wishart density that allows for, among other things, rotations of the extension. However, M3 requires a rotation parameter to be set, which can be difficult, especially in the multiple target case where the targets can maneuver with individual time varying turn-rates. The results show that the RMSEs increase when the parameter is set incorrectly.

In this comparison the multiple model framework M3 was implemented in two versions: one with the same three modes as were used in the simulation study in [10, Section IV], and one version with two additional rotation modes. An important issue to stress is that M3 can be implemented more flexibly, i.e. with more than 5 modes. A straightforward improvement would be to add additional rotation modes that have different probable $\theta$ values. Using a larger discrete set of parameter
TABLE I

MEAN CYCLE TIME \pm ONE ST.DEV. (ms)

\begin{tabular}{|lccc|}
\hline Method: & $\mathrm{M} 3 / 3$ & $\mathrm{M} 3 / 5$ & $\mathrm{M} 4$ \\
Cycle time: & $36 \pm 2$ & $84 \pm 2$ & $10 \pm 1$ \\
\hline
\end{tabular}

values, M3 would cover the unknown parameter space more efficiently. With M4, the measurements are used to estimate an individual turn-rate for each target, and in a sense M4 can be considered as a continuous parameter version of M3. However, note that in order to match M4, M3 might require a considerable number of modes, with a corresponding increase in computational demands. Table I gives the cycle times for M3/3, M3/5 and M4, averaged over all Monte Carlo runs. $\mathrm{M} 3 / 3$ is about 3.5 times slower than M4, and M3/5 is about 8.5 times slower. In this sense, M4 is more efficient than M3, because it handles a variable turn-rate using a single mode.

A final issue that must be mentioned for a fair comparison of M3 and M4 is that M3 uses Koch's random matrix model [7], while M4 uses Feldmann et al.'s random matrix model [8]. Due to this, it is not possible to make use of a CT model for M3. The ability to use such a CT model for M3 would enable one to obtain a good turn-rate estimate, which can be substituted into the multiple model framework of M3 to reduce its errors. This is essentially the idea that was used for model M2.

4) Multiple maneuvering targets: In [26], [27] a GIW version of the extended target PHD filter [28] is given. For prediction the standard method M1 is used. In the results section of [26] it is noted that multiple targets that move according to a $\mathrm{CV}$ model are easy to track with a $\mathrm{CV}$ motion model. However, targets that maneuver according to a CT model, while simultaneously being spatially close, are difficult to track. One problem is the simple CV prediction (7), which is insufficient to describe the target motion during maneuvers [26]. A result is that the filter cannot keep spatially close targets resolved during the maneuvers, resulting in cardinality being underestimated.

The presented prediction method M4 was used in the GIWPHD filter [26], and tested on a scenario with two targets. This scenario was also used in [26], and the true target tracks are shown in Figure 3c. While moving in parallel, the targets' 


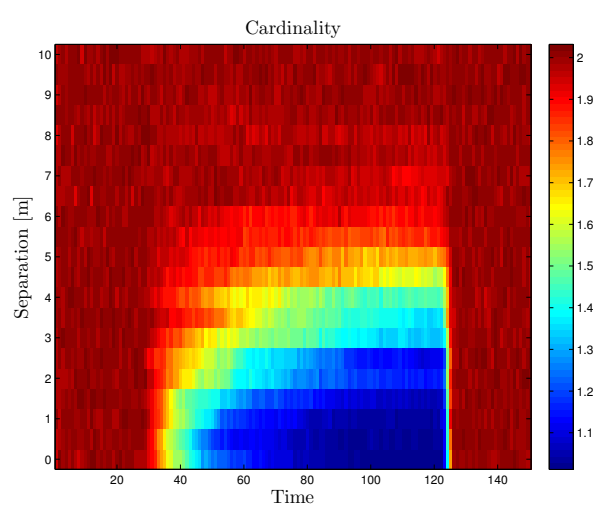

Fig. 6. Results for the multiple target scenario in Fig. 3c. At separation distances $d \geq 6 \mathrm{~m}$ the cardinality is estimated correctly.

extents were separated by a distance $d$. In [26] it was shown that the targets needed to be separated by $d \geq 21 \mathrm{~m}$ for the cardinality to be estimated correctly during the CT maneuver. At closer distance, the GIW-PHD filter could not keep the two targets resolved.

For this paper the scenario was simulated for separation $d=0,0.5,1, \ldots, 10[\mathrm{~m}]$. For each separation $d$, the scenario was simulated $N_{s}=100$ times. The mean estimated cardinality is shown in Figure 6. From the results we can make two observations. The first is that cardinality is estimated correctly for separation $d \geq 6 \mathrm{~m}$, which is an improvement over [26] where $d \geq 21 \mathrm{~m}$ was needed. This performance improvement is a direct result of using a prediction that allows for kinematic state dependent rotations of the extension estimate.

The second observation is that at separation $d \leq 4 \mathrm{~m}$, the cardinality is underestimated during $\mathrm{CV}$ motion (from time 40 to time 75 ), because the filter cannot keep the targets resolved. This is actually worse performance than [26], where the cardinality was estimated correctly during CV motion at separation $d=0 \mathrm{~m}$. The explanation is that the kinematic state motion model that is used in [26] is a better model for $\mathrm{CV}$ motion that the CT model used in this paper.

5) Summary of $2 D$ results: The results show that when the turn rate is known with high accuracy, the prediction methods M2, M3 and M4 perform similarly. However, when the turn rate is uncertain, M4 performs better because it estimates the turn rate directly from the measurement data, and it also considers the effects of turn rate uncertainty on the extension estimate. The scenario with two maneuvering targets shows that including rotation of the extension can significantly improve performance for multiple maneuvering target tracking.

\section{CONCLUDING REMARKS}

This paper presented a new prediction update for extended targets whose extensions are modeled as random matrices. The new prediction allows for transformation of the target extension during target maneuvers, and the main tools for deriving the prediction are presented in terms of three different theorems. Two of the theorems show how matrix variate probability density functions can be approximated, the third theorem shows how a conditional matrix variate distribution can be marginalized.

Results from simulating a single prediction step show that the presented prediction method outperforms related work in terms of the expected value, and covariance of the predicted extension. In two simulations with a single maneuvering target, it is shown that the presented prediction method is more general than related work, because it can estimate the turn rate online and does not rely on setting a parameter. In a simulation with two targets it was shown that the presented prediction method can improve performance significantly when the targets maneuver while being spatially close.

In future work, we plan to include the presented prediction method in a multiple model framework. This could include motion modes for, e.g., constant velocity motion, constant turn rate motion with rotation of the extension, and scaling of the extension. It would also be interesting to see how a scaling of the extension matrix could be made dependent on the kinematic state, possibly using a kinematic state that corresponds to the scaling rate. As noted in [7], [8], [10], scaling of the extension matrix has important applications for group target tracking.

This work has not considered the measurement update within the random matrix framework, see e.g. [10], [29] for some recent work on this topic. Coupling the extension measurement update to the turn rate could possibly improve estimation of the turn rate. Finally, in this work, we have used the random matrix model of Koch [7] and Feldmann et al. [8] which uses inverse Wishart densities to represent the target extents. A drawback of this methodology could be that in higher dimensions, a single parameter might not be sufficient to represent the uncertainty of the extent matrix. Hence, the consideration of more general matrix-variate densities, with many parameters to represent the uncertainty, might be necessary for high dimensions.

\section{ACKNOWLEDGMENT}

The authors would like to thank the Linnaeus research environment CADICS and the frame project grant Extended Target Tracking (621-2010-4301), both funded by the Swedish Research Council, as well as the project Collaborative Unmanned Aircraft Systems (CUAS), funded by the Swedish Foundation for Strategic Research (SSF), for financial support.

\section{REFERENCES}

[1] Y. Bar-Shalom and T. E. Fortmann, Tracking and data association, ser. Mathematics in Science and Engineering. San Diego, CA, USA: Academic Press Professional, Inc., 1987, vol. 179.

[2] D. J. Salmond and M. C. Parr, "Track maintenance using measurements of target extent," IEE Proceedings - Radar, Sonar and Navigation, vol. 150, no. 6, pp. 389-395, Dec. 2003.

[3] M. Baum and U. D. Hanebeck, "Random hypersurface models for extended object tracking," in IEEE International Symposium on Signal Processing and Information Technology (ISSPIT), Ajman, United Arab Emirates, Dec. 2009, pp. 178-183.

[4] K. Granström, C. Lundquist, and U. Orguner, "Tracking Rectangular and Elliptical Extended Targets Using Laser Measurements," in Proceedings of the International Conference on Information Fusion, Chicago, IL, USA, Jul. 2011, pp. 592-599. 
[5] C. Lundquist, K. Granström, and U. Orguner, "Estimating the Shape of Targets with a PHD Filter," in Proceedings of the International Conference on Information Fusion, Chicago, IL, USA, Jul. 2011, pp. 49-56.

[6] H. Zhu, C. Han, and C. Li, "An extended target tracking method with random finite set observations," in Proceedings of the International Conference on Information Fusion, Chicago, IL, USA, Jul. 2011, pp. 73-78.

[7] J. W. Koch, "Bayesian approach to extended object and cluster tracking using random matrices," IEEE Transactions on Aerospace and Electronic Systems, vol. 44, no. 3, pp. 1042-1059, Jul. 2008.

[8] M. Feldmann, D. Fränken, and J. W. Koch, "Tracking of extended objects and group targets using random matrices," IEEE Transactions on Signal Processing, vol. 59, no. 4, pp. 1409-1420, Apr. 2011.

[9] F. Lian, C.-Z. Han, W.-F. Liu, X.-X. Yan, and H.-Y. Zhou, "Sequential Monte Carlo implementation and state extraction of the group probability hypothesis density filter for partly unresolvable group targets-tracking problem," IET Radar, Sonar and Navigation, vol. 4, no. 5, pp. 685-702, Oct. 2010.

[10] J. Lan and X.-R. Li, "Tracking of extended object or target group using random matrix - part I: New model and approach," in Proceedings of the International Conference on Information Fusion, Singapore, Jul. 2012, pp. 2177-2184.

[11] A. K. Gupta and D. K. Nagar, Matrix variate distributions, ser. Chapman \& Hall/CRC monographs and surveys in pure and applied mathematics. Chapman \& Hall, 2000.

[12] R. E. Kalman, "A New Approach to Linear Filtering and Prediction Problems," Transactions of the ASME - Journal of Basic Engineering, vol. 82, no. Series D, pp. 35-45, Mar. 1960.

[13] X.-R. Li and V. Jilkov, "Survey of maneuvering target tracking: Part I. Dynamic models," IEEE Transactions on Aerospace and Electronic Systems, vol. 39, no. 4, pp. 1333-1364, Oct. 2003.

[14] S. Kullback and R. A. Leibler, "On information and sufficiency," The Annals of Mathematical Statistics, vol. 22, no. 1, pp. 79-86, Mar. 1951.

[15] J. L. Williams and P. S. Maybeck, "Cost-Function-Based Gaussian Mixture Reduction for Target Tracking," in Proceedings of the International Conference on Information Fusion, Cairns, Queensland, Australia, Jul. 2003.

[16] A. R. Runnalls, "Kullback-Leibler approach to Gaussian mixture reduction," IEEE Transactions on Aerospace and Electronic Systems, vol. 43, no. 3, pp. 989-999, Jul. 2007.

[17] D. Schieferdecker and M. F. Huber, "Gaussian Mixture Reduction via Clustering," in Proceedings of the International Conference on Information Fusion, Seattle, WA, USA, Jul. 2009.

[18] K. Granström and U. Orguner, "Properties and approximations of some matrix variate probability density functions," Department of Electrical Engineering, Linköping University, SE-581 83 Linköping, Sweden, Tech. Rep. LiTH-ISY-R-3042, Dec. 2011. [Online]. Available: http://urn.kb.se/resolve?urn=urn:nbn:se:liu:diva- 88735

[19] J. Stoer and R. Bulirsch, Introduction to Numerical Analysis, 2nd ed. New York: Springer-Verlag, 1993.

[20] C. M. Bishop, Pattern recognition and machine learning. New York, USA: Springer, 2006.

[21] T. Minka, "A family of algorithms for approximate Bayesian inference," Ph.D. dissertation, Massachusetts Institute of Technology, Jan. 2001.

[22] A. H. Jazwinski, Stochastic Processes and Filtering Theory. Academic Press, 1970.

[23] A. Gut, An Intermediate Course in Probability, 1st ed. New York, NY, USA: Springer, 1995.

[24] F. Gustafsson, L. Ljung, and M. Millnert, Signal Processing. Studentlitteratur, 2010.

[25] J. M. Ortega and W. C. Rheinboldt, Iterative Solution of Nonlinear Equations in Several Variables. Academic Press, 1970.

[26] K. Granström and U. Orguner, "A PHD filter for tracking multiple extended targets using random matrices," IEEE Transactions on Signal Processing, vol. 60, no. 11, pp. 5657-5671, Nov. 2012.

[27] — , "Implementation of the GIW-PHD filter," Department of Electrical Engineering, Linköping University, SE-581 83 Linköping, Sweden, Tech. Rep. LiTH-ISY-R-3046, Mar. 2012. [Online]. Available: http://urn.kb.se/resolve?urn=urn:nbn:se:liu:diva-94585

[28] R. Mahler, "PHD filters for nonstandard targets, I: Extended targets," in Proceedings of the International Conference on Information Fusion, Seattle, WA, USA, Jul. 2009, pp. 915-921.

[29] U. Orguner, "A variational measurement update for extended target tracking with random matrices," IEEE Transactions on Signal Processing, vol. 60, no. 7, pp. 3827-3834, Jul. 2012. 